\title{
Anisotropic Polymer Nanoparticles with Tunable Emission Wavelengths by Intersegmental Chain Packing
}

\author{
Julian D. Ruiz Perez and Stefan Mecking*
}

\begin{abstract}
The shape of nanoparticles is decisive for their selfassembly properties, as well as for the behavior of individual particles in many instances. The introduction and control of anisotropy is well-known for inorganic nanocrystals but a largely unresolved problem for polymer nanoparticles, especially for conjugated polymers. Here, we report a reproducible preparation method using heterophase polymerization for a range of stable dispersions of ellipsoidal conjugated polymer nanoparticles with sizes in the range of 50 to $180 \mathrm{~nm}$ and narrow size and shape distributions. The nanoparticles show a bright fluorescence with tunable emission wavelengths and quantum yields as high as $\varphi=78 \%$. Structural investigations reveal the nanoparticles to be composed of a highly ordered $\beta$ - and $\alpha^{\prime}$-phase within a nematic matrix. Preliminary studies on the origin of particle anisotropy suggest a concerted mechanism in which anisotropic shape evolves from intersegmental packing that occurs along with progressing chain formation by polymerization.
\end{abstract}

B eyond their chemical composition, the shape of nanoparticles has a tremendous impact on their properties. Wellknown examples are shape-dependent plasmon behavior ${ }^{[1]}$ or cellular internalization. ${ }^{[2]}$ While this concerns individual particles, also interparticle interactions are shape-dependent. Thus, the directional mechanical properties of nacre arise from packing of anisotropic particles. ${ }^{[3]}$ Different from inorganic nanocrystals, the accessibility of anisotropic polymer nanoparticles remains largely unexplored. This applies in particular to conjugated polymer nanoparticles (CPNs) as an important class of materials.

CPNs are studied broadly due to their favorable photoand electroluminescent properties. Applications comprise live cell imaging, biosensing, and optoelectronics. ${ }^{[4]}$ The ability to adjust their emission wavelengths is decisive here. ${ }^{[9]}$

Commonly, CPNs are prepared by post-polymerization dispersion techniques, ${ }^{[5,6,10]}$ or also by heterophase polymerizations. ${ }^{[7,8,11,12]}$ Both approaches have in common that, with very few exceptions, ${ }^{[10,11]}$ exclusively spherical CPNs are obtained.

Known procedures to generate anisotropic polymer particles rely on methods for post-polymerization shaping of

[*] M. Sc. J. D. Ruiz Perez, Prof. Dr. S. Mecking

Chair of Chemical Materials Science, Department of Chemistry

University of Konstanz

Universitätsstrasse 10, 78457 Konstanz (Germany)

E-mail: stefan.mecking@uni-konstanz.de spherical particles, like electrified jetting, ${ }^{[13]}$ microcontact printing,${ }^{[14]}$ emulsion drying, ${ }^{[15]}$ surface templating, ${ }^{[16]}$ lithographic methods ${ }^{[17]}$ as well as stretching of films containing spherical CPNs. ${ }^{[18]}$ A drawback of these methods is the limitation to micrometer-sized structures as well as the lack of scalability. Concepts to introduce and control shape anisotropy directly in dispersion are missing, in particular on the nm scale.

We now report a reproducible protocol for the generation of ellipsoidal conjugated polymer nanoparticles and provide first insight into the origin of anisotropy. The utility and scope of the method are probed by the incorporation of fluorescent dyes in the polymer backbone, simultaneously enabling control of the emission wavelength.

As a starting point, in heterophase polymerizations to conjugated polymer nanoparticles, we occasionally observed non-spherical particles in different protocols. ${ }^{[7,12,19]}$ This was also the case for Suzuki-Miyaura coupling polymerization to produce both, particles of homopolymers of poly(9,9-dioctylfluorene) (PF8) and related copolymers (Scheme 1). Towards the aim of obtaining non-spherical particles selectively, parameters anticipated being relevant namely the nature of

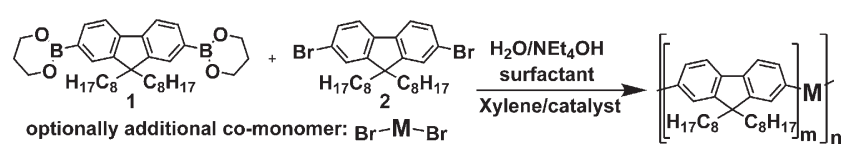

Scheme 1. General reaction scheme for Suzuki-Miyaura coupling polymerizations.

the monomers, the total reaction volume, the concentration of xylene and the type and concentration of surfactant were explored. As a measure of anisotropy and shape homogeneity of the obtained CPNs, the mean values of length and aspect ratio (ar) as observed by TEM analysis were calculated and the coefficient of variation $v$ was determined in either case (Table 1 and Table S1 in the Supporting Information).

From this empirical study of the above parameters suitable conditions evolved for the heterophase polymerization of the diboronic acid ester $\mathbf{1}$ and the dibromosubstituted monomer $\mathbf{2}$ (Figure 1) to exclusively one kind of particles with a well-defined ellipsoidal shape, a high aspect ratio of 3.6 and a high shape homogeneity (cf. Table 1, entry c, $v_{\text {length }}=0.12$ and $v_{\mathrm{ar}}=0.13$, Figure 2 ). Cryo-TEM proved that the ellipsoidal nanoparticles indeed exist in dispersion and do not form during the preparation of the TEM samples. Notably, independent repetitive experiments under identical conditions underlined the reproducibility and reliability of this method (Table S3). Furthermore, these experiments show 
Table 1: Heterophase Suzuki-Miyaura coupling polymerizations under different conditions to produce anisotropic CPNs. ${ }^{[\mathrm{a}]}$

\begin{tabular}{cccccc}
\hline Entry & Xylene $[\mathrm{mL}]$ & Surfactant & $V[\mathrm{~mL}]$ & \multicolumn{2}{c}{ TEM } \\
& & & & length $[\mathrm{nm}]^{[\mathrm{b}]} / \nu$ & $\operatorname{ar}^{[\mathrm{c}]} / \nu$ \\
\hline a & 2 & Triton X-102 & 50 & $46 / 0.20$ & $2.3 / 0.26$ \\
$\mathrm{~b}$ & 2 & Triton X-102 & 25 & $100 / 0.15$ & $2.8 / 0.14$ \\
c & 1 & Triton X-102 & 25 & $112 / 0.12$ & $3.6 / 0.13$ \\
d & 1 & Tween 80 & 25 & $48 / 0.30$ & $1.3 / 0.15$ \\
$\mathrm{e}^{[\mathrm{d}]}$ & 1 & Triton X-102 & 25 & $178 / 0.19$ & $3.1 / 0.21$ \\
$\mathrm{f}^{\text {fe] }}$ & 1 & Triton X-102 & 25 & $87 / 0.22$ & $1.2 / 0.11$ \\
\hline
\end{tabular}

[a] Reaction conditions: Monomers dissolved in xylene, $5 \mathrm{wt} \%$ aqueous surfactant solution, 1 equiv tetraethylammonium hydroxide, $2 \mathrm{~mol} \%$ $\left[\mathrm{Pd}\left(\mathrm{P}^{\mathrm{t}} \mathrm{Bu}_{3}\right)_{2}\right]$ catalyst, $48 \mathrm{~h}$. [b] Mean value of length and [c] mean aspect ratio (ar) and coefficient of variation $v$ in either case. [d] Twofold concentration of monomers, base and catalyst. [e] $\left[\mathrm{Pd}\left(\mathrm{PPh}_{3}\right)_{4}\right]$ was used as a catalyst precursor.
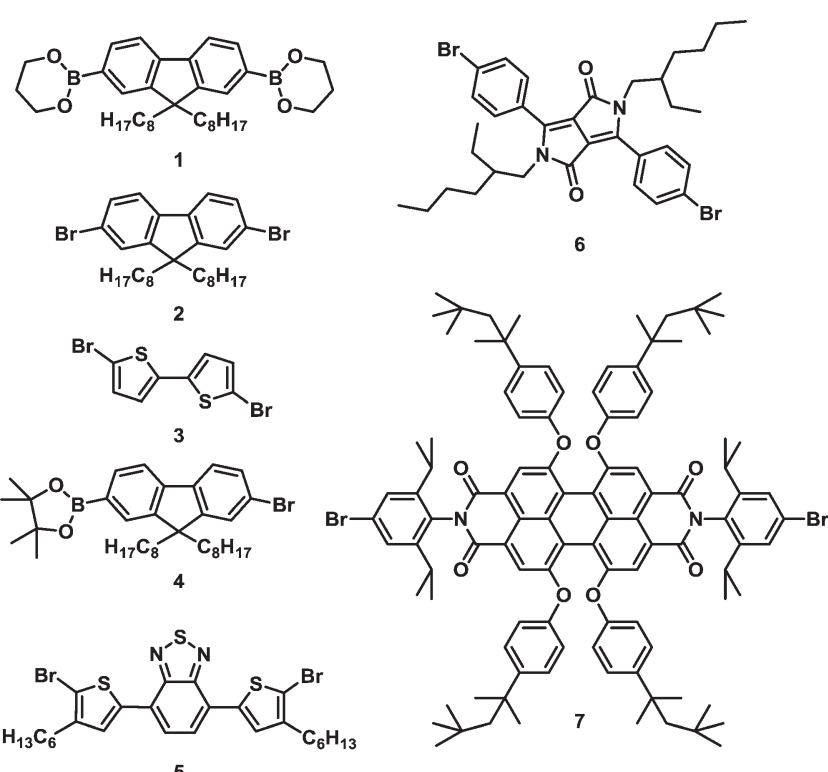

Figure 1. Monomers employed in Suzuki-Miyaura coupling polymerization.

that particle shape and size are rather independent of the molecular weight of the polymer. It is also possible to control the size of the ellipsoidal CPNs. Using a twofold concentration of monomers, base, and catalyst under otherwise identical conditions provided larger ellipsoids (Figure S16), with a slightly lower shape homogeneity (cf. Table 1, entry e, $v_{\text {length }}=0.19$ and $v_{\mathrm{ar}}=0.21$ ). The versatility of this approach was shown by the preparation of anisotropic CPNs based on the AB-monomer $\mathbf{4}$ as well as the combination of monomer 1 with 5,5'-dibromo-2,2'-bithiophene (3) (cf. Table S1).

Concerning the impact of reaction conditions, the total volume of the aqueous phase has a strong effect on particle shape and shape homogeneity (cf. Table 1, entries a,b). By contrast, the amount of xylene solvent hardly affects particle shape (cf. Table 1, entries b,c and see the Supporting Information). This suggests that an introduction of sufficient shear energy to the initial reaction mixture is critical.

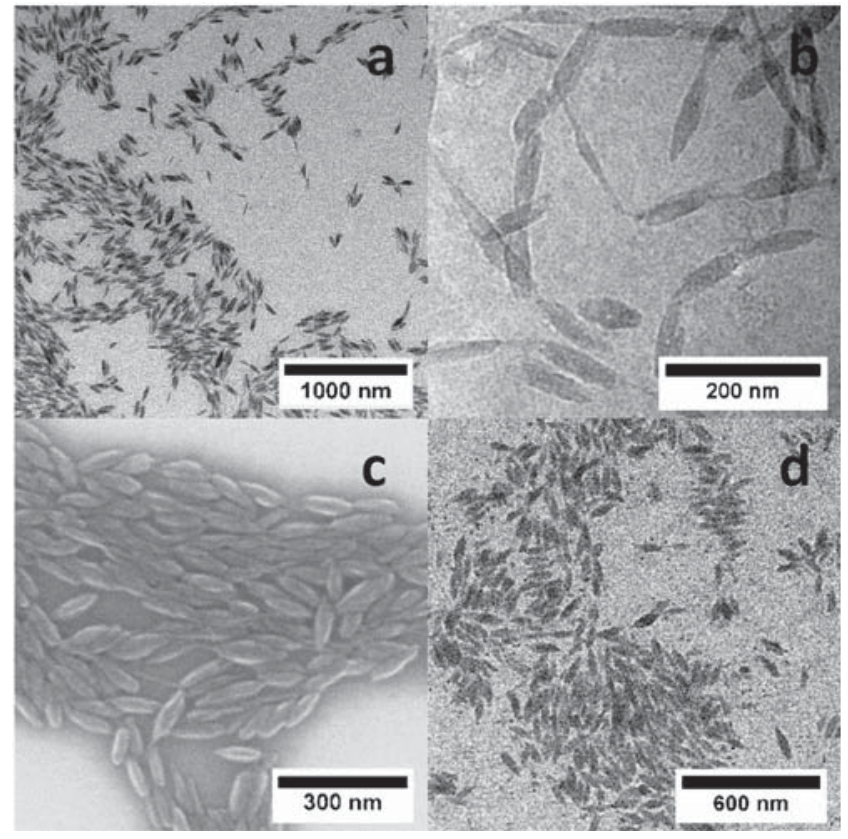

Figure 2. a) TEM, b) cryo-TEM, and c) SEM images of homopolymer dispersion and d) TEM image of a copolymer dispersion with $0.5 \mathrm{~mol} \%$ of 5 .

The nature of the surfactant is found to be equally decisive. The utilization of Triton X-100 instead of Triton X102, with a slightly shorter PEG-chain (9-10 vs. 12-13 repeat units, with a $p$-\{1,1,3,3-tertra(methyl)butyl\}phenyl hydrophobic motif in both cases), only yields a small amount of ellipsoidal particles (cf. Table S1, ar=2.0). A more drastic variation, using the nonionic surfactant Tween 80 (polyoxyethylene(20)-sorbitan-monooleat), solely gives spherical CPNs (cf. Table 1, entry d, ar=1.3). Concerning the concentration of Triton X-102 surfactant, moderate variation did not impact the homogeneity of particle shape significantly. Likewise, the procedure appears to be relatively insensitive to reaction temperature (cf. Table S1).

By contrast, the choice of catalyst was found to be decisive for obtaining anisotropic CPNs of high shape homogeneity, and high polymer molecular weights. Utilization of the common catalyst precursor $\left[\mathrm{Pd}\left(\mathrm{PPh}_{3}\right)_{4}\right]$ instead of $\left[\mathrm{Pd}\left(\mathrm{P}^{t} \mathrm{Bu}_{3}\right)_{2}\right]$ led exclusively to spherical CPNs (aspect ratio of 1.2), as also reported previously (cf. Table 1 , entry f).$^{[8]}$ $\left[\mathrm{Pd}\left(\mathrm{P}^{\mathrm{t}} \mathrm{Bu}_{3}\right)_{2}\right]$ shows an increased reaction rate, ${ }^{[20,21]}$ compared to $\left[\mathrm{Pd}\left(\mathrm{PPh}_{3}\right)_{4}\right]$, which leads to a faster formation of the $\beta$ phase as evidenced by fluorescence emission spectroscopy monitoring of the polymerization reaction (Figure 3 ). For the $\left[\mathrm{Pd}\left(\mathrm{P}^{\mathrm{t}} \mathrm{Bu}_{3}\right)_{2}\right]$-system the emission peak at $416 \mathrm{~nm}$, which corresponds to the glassy phase, was already low in intensity after one day and completely disappeared after four days of reaction time, while the observed main peaks at 438,464 , and $496 \mathrm{~nm}$ can be assigned to the $\beta$-phase. In the case of the $\left[\mathrm{Pd}\left(\mathrm{PPh}_{3}\right)_{4}\right]$-system the proportion of the $\beta$-phase only increased slowly. A sufficiently rapid polymerization and correspondingly rapid formation of $\beta$-phase appear crucial for the formation of anisotropic, ellipsoidal CPNs. 


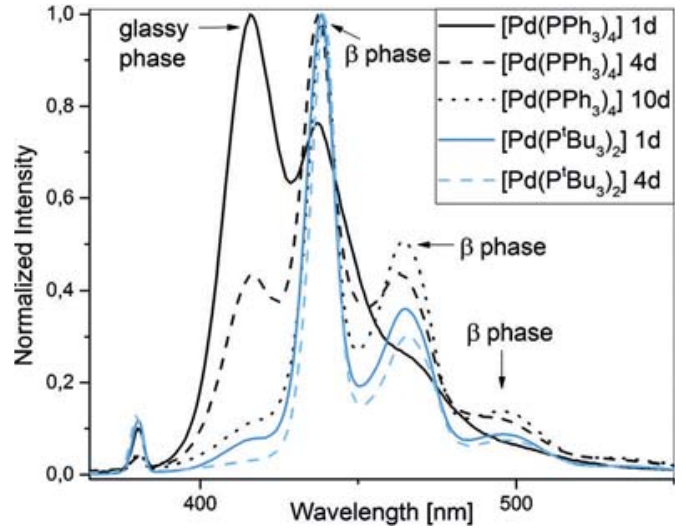

Figure 3. Comparison of emission spectra of dispersions prepared using $\left[\mathrm{Pd}\left(\mathrm{PPh}_{3}\right)_{4}\right]$ or $\left[\mathrm{Pd}\left(\mathrm{P}^{\mathrm{t}} \mathrm{Bu}_{3}\right)_{2}\right]$ at different reaction times.

The prepared anisotropic CPNs feature a bright fluorescence in the blue regime arising from the PF8 backbone. An emission at higher wavelength up to the deep red regime would be desirable. In biological imaging, for example, this results in less disturbing background fluorescence. ${ }^{[22]}$

To this end, a covalent incorporation of fluorescent dyes during the polymerization has been demonstrated to be a powerful tool. ${ }^{[7,23]}$ However, assuming an intersegmental packing within the particles being responsible for the ellipsoidal shape, a variation of the polymer backbone is expected to affect the anisotropic shape. In a first approach the dithiophene-substituted N,S-heterocycle $\mathbf{5}$ was employed. The heterophase terpolymerizations of the boronic ester $\mathbf{1}$ with a stoichiometric amount of the dibromides $\mathbf{2}$ and $\mathbf{5}$ yielded dispersions with a varying content of $\mathbf{5}$ between 0.5 and $5 \mathrm{~mol} \%$ (Table S2). UV/Vis absorption spectra revealed the formation of $\beta$-phase (around $436 \mathrm{~nm}$ ) in the formed nanoparticles as well as an absorption maximum around $405 \mathrm{~nm}$, very similar to the UV/Vis spectrum of the dispersion of the homopolymer (Figure 4, top). The absorbance of the fluorene-dye segments is observed as a broad shoulder that partially overlaps with the $\beta$-phase absorption, extends to around $600 \mathrm{~nm}$ and accounts for the reddish appearance of the dispersions (Figure 4, top, inset). As expected, the absorption intensity between $450-650 \mathrm{~nm}$ increases with increasing amount of incorporated dye 5. Similarly, the fluorescence emission spectra show an increasing emission in the red regime, $550-850 \mathrm{~nm}$, besides the well-known emission of the PF8 $\beta$-phase ${ }^{[24,25]}$ (Figure 4, bottom).

Comparing the quantum yield of the homopolymer nanoparticles (35\%), which is similar to values reported for spherical PF8 CPNs prepared by other methods, ${ }^{[25]}$ the copolymer nanoparticles feature much higher quantum yields above $70 \%$. These high quantum yields remain virtually unaltered with increasing amount of incorporated dye. In contrast, the shape homogeneity diminished noticeably, resulting in a heterogeneous distribution of particle size and shape at high dye incorporation. Thus, there is indeed a relation between backbone imperfections and shape homogeneity, indicating the significance of an internal order. However, for the dispersions with low incorporations of 0.5 and $1 \mathrm{~mol} \%$, respectively, well-defined ellipsoids were
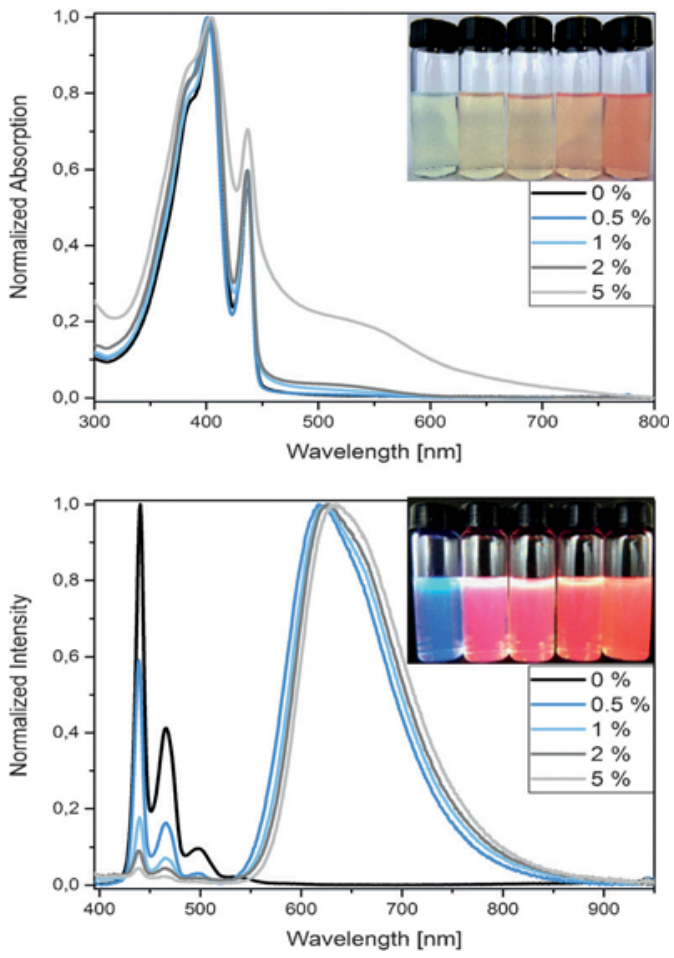

Figure 4. Absorption spectra (top) and emission spectra (bottom) of comonomer dispersions with a varying content of $\mathbf{5}$ between 0 and $5 \mathrm{~mol} \%$. The inserts show the appearance of the 100-fold diluted dispersions in daylight (top) and under UV-light irradiation (bottom).

obtained, comparable to those of the homopolymer dispersion, while at the same time the emission color is fully shifted to red (Figure 2d).

Ellipsoidal particles with a physically incorporated dye could be generated by polymerization of $\mathbf{1}$ and $\mathbf{2}$ in the presence of a non-polymerizable analog of $\mathbf{5}$. Whereas shape homogeneity and quantum yields are comparable to the copolymer particles, the covalent incorporation in the latter lead to an increased shift into the far-red due to the extended $\pi$-system (see the Supporting Information).

In order to demonstrate the general scope of the method, two further comonomers $(\mathbf{6}, \mathbf{7})$ were used to prepare dispersions with tuned emission spectra. Whereas the UV/ Vis spectra do not reveal any major differences compared to the homopolymer dispersion, the fluorescence emission spectra are dominated by local emission maxima at 572 and $603 \mathrm{~nm}$, respectively (Figure S3). TEM images again reveal well-shaped ellipsoidal CPNs (Figure 5). Consequently, all copolymer particles with a dye content of $0.5 \mathrm{~mol} \%$ are still highly anisotropic $(\operatorname{ar}>3)$ and homogenous $(v \leq 0.13)$. It is remarkable that even the bulky perylendiimide comonomer 7 does not disturb the formation of ellipsoidal CPNs, at least for a low incorporation of $0.5 \mathrm{~mol} \%$.

At this point, the origin of particle anisotropy remains unclear. Evidently, the polymerization procedure is critical, although anisotropic particles can be obtained reliably over a range for different parameters. By broad analogy to inorganic nanocrystals and polymer nanocrystals, an intermolecular packing was considered to be involved. 


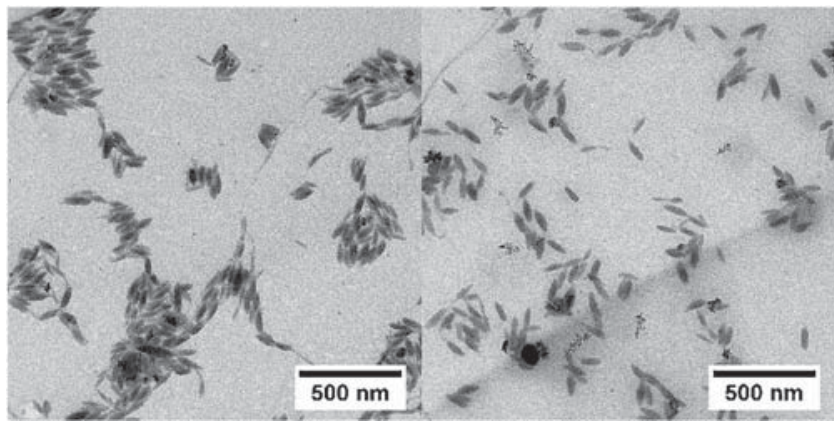

Figure 5. TEM images of dispersions with incorporated monomer 6 (left) and 7 (right).

The effect of the choice of catalyst and of the nature of the (co)monomer already suggested that the chain conformation and packing, as reflected by the proportion of $\beta$-phase, is a key to the particle shape for the present system.

To this end, PXRD studies were conducted on polymer precipitated from the dispersion by adding an excess of methanol. The diffractograms of all polymers show two characteristic peaks centered around $2 \theta=6.9^{\circ}$ and $21.7^{\circ}$ (Figure S4). By comparison to values obtained from PF8 films, these peaks can be assigned to crystalline domains composed of $\alpha^{\prime}$-phase, which possesses an oriented nature. ${ }^{[26]}$

Additionally, the absorption and fluorescence emission spectra of the comonomer dispersions all show peaks which can be unambiguously assigned to the $\beta$-phase (Figure 4, S3). The CPNs are semicrystalline with an amorphous portion, as obvious from the broad halo in all shown diffractograms. ${ }^{[6]}$ Thus, the CPNs appear to be composed of the $\alpha^{\prime}$ - and $\beta$-phase within a nematic matrix. These highly ordered phases with a rigid conformation of the polyfluorene backbone are presumably responsible for the evolution of an anisotropic shape (cf. Figure 6).

To further understand the formation of anisotropic particle shapes, a reaction mixture without catalyst was prepared. Ultrasonication for two minutes analogous to the above heterophase polymerization protocol yielded a clear, colorless sample (note that when stirring with a magnetic stir bar, without ultrasonication no emulsification occurred and a two-phase system was formed). Other than in a polymerization experiment, where turbidity sets in shortly after emulsification, the ultrasonicated emulsion without catalyst stays clear. This suggests the ongoing polymerization already in its early stages alters the droplet or particle, respectively, with regard to its size.
To gain more insight into the processes occurring after emulsification, the monomers were ultrasonicated separately in a water/base mixture forming two monomer emulsions. These were mixed subsequently. Although no additional shear forces were applied, polymerization occurred. This observation shows that exchange of material occurs (like in a microemulsion ${ }^{[27]}$, rather than a preservation of the initial monomer droplets' individual identity.

Taking all findings into account a preliminary mechanism of particle formation is suggested (Figure 6). First, during ultrasonication ( 2 minutes) a clear emulsion forms consisting of small monomer droplets (around 10-20 nm). Right after, driven by the onset of polymer formation, these droplets increase in size (and their number decreases), which results in the onset of turbidity of the emulsion (Figure 6 A). Along with the ongoing polymerization, an alignment of the polymer chains occurs (Figure 6B), resulting in the formation of CPNs with an ellipsoidal shape. Over several hours the xylene that swells the nanoparticles is lost and anisotropic, blue fluorescent PF8 nanoparticles are obtained (Figure 6C). Obviously, the individual steps (namely polymerization chain growth, orientation, particle formation and xylene diffusion) do not occur one after the other, but rather in a concerted fashion.

In summary, we reveal a robust method for direct access to anisotropic conjugated polymer nanoparticles with an ellipsoidal shape via heterophase polymerization. The reaction volume and energy input, the type of surfactant as well as the choice of catalyst were identified as the most decisive parameters in terms of quantity, shape and homogeneity of the anisotropic CPNs. Noteworthy, the formation of anisotropic ellipsoidal particles is not limited to a narrow range or a very singular combination of parameters and the protocol is highly reproducible concerning particle shape and dimensions. Thus, the emission properties and color of the resulting aqueous dispersions can be tuned without loss of the anisotropic particle shape via incorporation of electronwithdrawing repeat units.

Absorption and photoluminescence spectroscopy, as well as powder X-ray diffraction, confirm the semicrystalline character of the CPNs. Preliminary studies on the origin of particle anisotropy suggest a concerted mechanism in which anisotropic shape evolves from interchain packing that occurs along with progressing chain formation by polymerization. Consequently, particle formation proceeds in a kinetically controlled fashion. The anisotropic CPNs now available are attractive as building blocks for particle-based polymer (hybrid) materials with potentially directional properties.
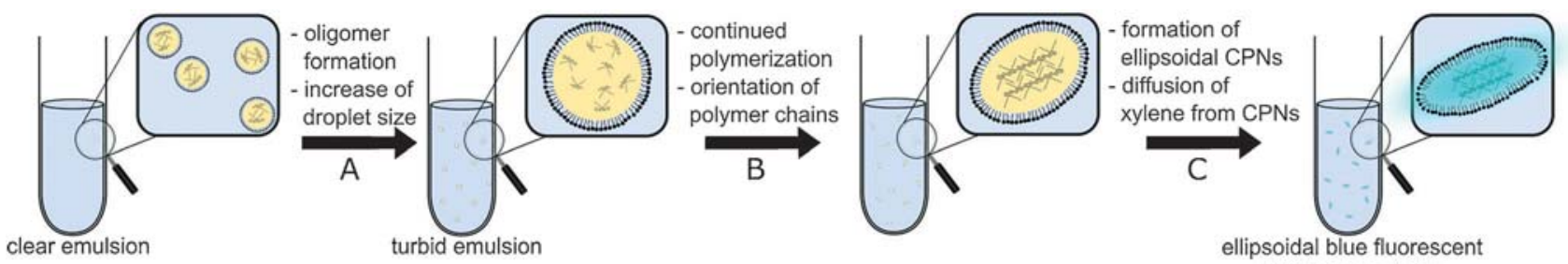

ellipsoidal blue fluorescent PF8 nanoparticles

Figure 6. Proposed pathway of nanoparticle formation. 


\section{Experimental Section}

Procedure for heterophase polymerization: In a $100 \mathrm{~mL}$ Schlenk tube, tetraethylammonium hydroxide solution $(20 \%$ in water $)$ $(0.16 \mathrm{~g}, 0.2 \mathrm{mmol})$ was added to an aqueous solution $(25 \mathrm{~mL})$ of Triton X-102 nonionic surfactant ( $1.25 \mathrm{~g}, 5 \mathrm{wt} \%$ in deionized water). The mixture was sparged for 30 minutes with nitrogen gas under stirring with a stir bar. In a $10 \mathrm{~mL}$ Schlenk tube the monomers $(0.1 \mathrm{mmol}$ each) were dissolved in xylene $(1 \mathrm{~mL})$, the solution was degassed by two freeze-pump-thaw cycles, and transferred to another $10 \mathrm{~mL}$ Schlenk tube containing the catalyst precursor $(2.1 \mathrm{mg}$, $4 \mu \mathrm{mol}$ ). The solution was again degassed by two freeze-pumpthaw cycles, and injected via a syringe into the aqueous surfactant/ base solution and emulsified by an ultrasonotrode (Bandelin GM3200 with KE76 tip, operated at $120 \mathrm{~W}$ ) for two minutes. The contents were stirred under nitrogen gas at room temperature for at least $48 \mathrm{~h}$.

\section{Acknowledgements}

Financial support by the DFG (SFB1214) and by the Center of Applied Photonics is gratefully acknowledged. We thank L. Bolk for GPC and M. Krumova for SEM measurements.

\section{Conflict of interest}

The authors declare no conflict of interest.

Keywords: conjugated polymers · cross-coupling · fluorescence $\cdot$ nanoparticles $\cdot$ polymerization

[1] N. J. Halas, Nano Lett. 2010, 10, 3816-3822.

[2] S. E. A. Gratton, P. A. Ropp, P. D. Pohlhaus, J. C. Luft, V. J. Madden, M. E. Napier, J. M. DeSimone, Proc. Natl. Acad. Sci. USA 2008, 105, 11613-11618.

[3] C. M. Zaremba, A. M. Belcher, M. Fritz, Y. Li, S. Mann, P. K. Hansma, D. E. Morse, J. S. Speck, G. D. Stucky, Chem. Mater. 1996, 8, 679-690.

[4] J. Pecher, S. Mecking, Chem. Rev. 2010, 110, 6260-6279.

[5] K. Landfester, R. Montenegro, U. Scherf, R. Güntner, U. Asawapirom, S. Patil, D. Neher, T. Kietzke, Adv. Mater. 2002, 14, 651-655.
[6] C. Wu, B. Bull, C. Szymanski, K. Christensen, J. McNeill, ACS Nano 2008, 2, 2415-2423.

[7] M. C. Baier, J. Huber, S. Mecking, J. Am. Chem. Soc. 2009, 131, $14267-14273$.

[8] R. Wang, C. Zhang, W. Wang, T. Liu, J. Polym. Sci. Part A 2010, 48, $4867-4874$.

[9] C. F. Huebner, S. H. Foulger, Langmuir 2010, 26, 2945-2950.

[10] Z. Yang, W. T. S. Huck, S. M. Clarke, A. R. Tajbakhsh, E. M. Terentjev, Nat. Mater. 2005, 4, 486-490.

[11] D. Muenmart, A. B. Foster, A. Harvey, M.-T. Chen, O. Navarro, V. Promarak, M. C. McCairn, J. M. Behrendt, M. L. Turner, Macromolecules 2014, 47, 6531-6539.

[12] J. Pecher, S. Mecking, Macromolecules 2007, 40, 7733 - 7735.

[13] K.-H. Roh, D. C. Martin, J. Lahann, Nat. Mater. 2005, 4, 759763.

[14] O. Cayre, V. N. Paunov, O. D. Velev, J. Mater. Chem. 2003, 13, $2445-2450$

[15] V. N. Manoharan, M. T. Elsesser, D. J. Pine, Science 2003, 301, $483-487$.

[16] J. P. Rolland, B. W. Maynor, L. E. Euliss, A. E. Exner, G. M. Denison, J. M. DeSimone, J. Am. Chem. Soc. 2005, 127, 1009610100.

[17] D. Dendukuri, D. C. Pregibon, J. Collins, T. A. Hatton, P. S. Doyle, Nat. Mater. 2006, 5, 365-369.

[18] A. J. C. Kuehne, M. C. Gather, J. Sprakel, Nat. Commun. 2012, 3, 1088.

[19] C. Negele, J. Haase, A. Leitenstorfer, S. Mecking, ACS Macro Lett. 2012, 1, 1343-1346.

[20] K. H. Shaughnessy, P. Kim, J. F. Hartwig, J. Am. Chem. Soc. 1999, 121, 2123-2132.

[21] A. F. Littke, G. C. Fu, J. Org. Chem. 1999, 64, 10-11.

[22] C. Wu, D. T. Chiu, Angew. Chem. Int. Ed. 2013, 52, 3086-3109; Angew. Chem. 2013, 125, 3164-3190.

[23] C. S. Fischer, M. C. Baier, S. Mecking, J. Am. Chem. Soc. 2013 , $135,1148-1154$.

[24] S. H. Chen, A. C. Su, S. A. Chen, J. Phys. Chem. B 2005, 109, $10067-10072$.

[25] C. Wu, J. McNeill, Langmuir 2008, 24, 5855-5861.

[26] S. H. Chen, A. C. Su, C. H. Su, S. A. Chen, Macromolecules 2005, 38, 379-385.

[27] D. J. McClements, Soft Matter 2012, 8, 1719-1729. 